cologique d'hypolipémiants particulièrement prometteuse. Ces molécules ont démontré leur activité in vivo dans un modèle animal et in vitro sur des hépatocytes humains. II sera donc nécessaire de confirmer leur activité chez l'homme avant d'envisager cette nouvelle classe pharmacologique comme une alternative thérapeutique. $\diamond$

A new family of drugs lowering LDL
RÉFÉRENCES

1. Ansell BJ, Watson $K \varepsilon$, Fogelman AM. An evidencebased assessment of the NCEP adult treatment panel II guidelines. National cholesterol education program. JAMA 1999 ; 282 : 2051-7.

2. Kovanen PT, Schneider WJ. Regulation of the low density lipoprotein $(B / \varepsilon)$ receptor. Adv Vasc Biol 1999 ; 5 : 165-85.

3. Hua X, Nohturfft A, Goldstein JL, Brown MS.
Protein sterol resistance in $\mathrm{CHO}$ cells traced to point mutation in SREBP cleavage-activating protein. Cell 1996 ; 87 : 415-22.

4. Matsuda M, Korn BS, Hammer RE, et al. SREBP cleavage-activating protein (SCAP) is required for increased lipid synthesis in liver induced by cholesterol deprivation and insulin elevation. Genes Dev 2001 ; 15 : 1206-16.

5. Sakai J, Nohturfft A, Goldstein JL, Brown MS. Cleavage of sterol

\title{
NOUVELLE
}

\section{La titine retrouvée en pathologie cardiaque}

Simone Gilgenkrantz

Simone Gilgenkrantz,

$>$ Dans l'unité contractile du muscle, la titine joue un rôle essentiel. Encore appelée connectine, cette protéine géante (3 000 kDa environ) peut être comparée à une sorte de collier élastique d'environ 300 perles (les domaines fibronectine de type III : FN3, et les domaines immunoglobuline : Ig) qui s'étend, au sein d'un hémi-sarcomère, de la ligne $M$ (point d'ancrage des filaments de myosine) à la ligne $Z$ (point d'ancrage des filaments d'actine) (Figure 1). Elle coordonne le parfait alignement des myofibrilles dans le muscle strié et confère à celui-ci $(\rightarrow) m / s \quad$ son élasticité $(\rightarrow)$. 1997, n¹0, Depuis 1995 , on connaît p.1189 la différence entre les isoformes du muscle squelettique et celles du myocarde [1]. Il va sans dire que la recherche des gènes impliqués dans les cardiomyopathies génétiques n'avaient pas exclu la titine. Toutefois, parmi les nombreux gènes découverts dans les cardiomyopathies
9 rue Basse, 54330

hypertrophiques (CMH), le gène TTN, codant pour Clerey/Brenon, France.

\section{cette énorme protéine,}

faisait pâle figure : sur une centaine de cas étudiés, une seule mutation (il s'agissait d'une transversion Arg 740 à Leu) avait été découverte chez un malade japonais [2].

Mais voici que, tout récemment, deux études, l'une chez le poisson-zèbre, l'autre chez l'homme, sont venues souligner la responsabilité de la titine dans les cardiomyopathies dilatées (CMD).

Les maux de cœur du poisson zèbre médecine/sciences a déjà évoqué l'intérêt de l'analyse des innombrables mutations provoquées chez le poisson zèbre (Danio rerio), ainsi que le recours aux « morpholinos » (oligonucléotides chimiquement transformés pouvant bloquer la traduction de l'ARNm). Ils accélèrent l'analyse fonctionnelle des gènes $(\rightarrow \rightarrow)$. La mutation Pik (pour pickwick, sans doute en raison de l'as- regulatory element-binding proteins (SREBPs) at site-1 requires interaction with SREBP cleavage-activating protein. Evidence from in vivo competition studies. J Biol Chem 1998 ; 273 : 5785-93.

6. Briggs MR, Yokoyama C, Wang X, Brown MS, Goldstein JL. Nuclear protein that binds sterol regulatory element of low density lipoprotein receptor promoter. J Biol Chem 1993 ; 268 : 14490-6.

7. Grand-Perret T, Bouillot A, Perrot A, Commans $S$, Walker M, Issandou M. SCAP ligands are potent new lipid-lowering drugs. Nat Med $2001 ; 7: 1332-8$.
Fect obèse des poissons,
dû à l'œdème consécutif au dysfonctionnement cardiaque), vient de trouver son explication moléculaire [3]. Les poissons Pikmlil ont un cœur morphologiquement normal, mais qui, dès le premier battement, semble très peu contractile. Le phénotype de ces mutants est par ailleurs normal pendant les trois premiers jours de vie, sans atteinte des muscles squelettiques. Par clonage positionnel, les auteurs ont tout d'abord observé que la région de $1,2 \mathrm{cM}$, où se trouvait la mutation, chevauchait le locus du gène ttn (codant pour la titine). En raison de l'intégrité du tissu musculaire squelettique, les auteurs supposèrent l'existence d'une mutation dans une isoforme propre au myocarde. Chez les rongeurs et chez l'homme, on savait qu'un domaine N2B n'existait que dans la protéine myocardique. Ce $(\rightarrow \rightarrow) m / s$ 2002, nº, p. 448 
domaine avait du reste été exploré, mais sans succès, dans une grande famille amérindienne porteuse de cardiomyopathie dilatée [4]. En séquençant ce domaine chez le poisson-zèbre, une transversion $T \rightarrow G$ fut trouvée dans une séquence unique, le domaine IS3, de l'exon N2B (Figure 2). Pour faire la preuve du rôle pathogène de cette mutation, deux oligonucléotides morpholinos furent construits : MOl qui cible le site donneur d'épissage de la terminaison 3' de l'exon contenant N2B, et M02 pour le site d'épissage de l'exon spécifique du muscle squelettique. Seule l'injection de MOl entraîne un phénotype identique à celui des mutants $P i k^{m 171}$. Une étude comparative de la formation des cardiomyocytes chez les poissons sauvages et chez les mutants montre que l'organisation des filaments épais et des filaments fins pour former des myofibrilles se fait de façon identique à 36 heures post-fécondation (hpf). Mais, alors que les sarcomères se forment à 48 hpf chez les témoins, ils n'apparaissent pas chez les mutants. Chez eux, les cardiomyocytes sont très fins et, lorsque des cœurs-mosaïque sont créés, les cellules pik survivent, mais font saillie, formant des anévrismes qui ne s'étendent pas aux cellules normales. II s'agit donc bien d'une action cellule-autonome. Ce rôle essentiel de la titine dans la formation des sarcomères vient confirmer d'autres études faites sur les cardiomyocytes de rat démontrant que la perte de l'intégrité de la titine provoque une diminution de cette « sarcomérogenèse ». Quant à la relaxation diastolique, elle est due à l'élasticité de trois domaines de la bande I: domaine Ig, PEVK et N2B. $[5,6]$. Du point de vue fonctionnel, chez le poisson zèbre, la relaxation diastolique et la pression systolique sont diminuées, de même que la fraction d'éjection, le cœur dilaté se vidant très peu au cours de la systole, comme il advient chez les malades atteints de cardiomyopathie dilatée.
Cardiomyopathies dilatées causées par des mutations de la titine

À l'instar des cardiomyopathies hypertrophiques, de très nombreux locus ont été trouvés dans les cardiomyopathies dilatées (ce qui permet un classement), mais peu de gènes ont encore été identifiés. Dans deux familles non apparentées porteuses d'une anomalie, dont le locus se situait en 2q31 (CMDIG), l'analyse de 313 des 363 exons de TTN exprimés dans le muscle cardiaque, a permis de découvrir deux mutations [7]. L'une est une insertion de deux pb dans l'exon 236 engendrant un codon stop prématuré. Une étude de la molécule tronquée, faite à l'aide d'anticorps préparés pour les différents segments de la titine, démontre l'intégrité de la région amino-terminale. L'autre est une substitution d'un tryptophane (très conservé) par une arginine en position 930, dans le premier domaine Ig, dans la zone de transition bande I - ligne Z (correspondant à l'exon 18) (Figure 3). Curieusement, ces mutations ne se trouvent pas dans une région

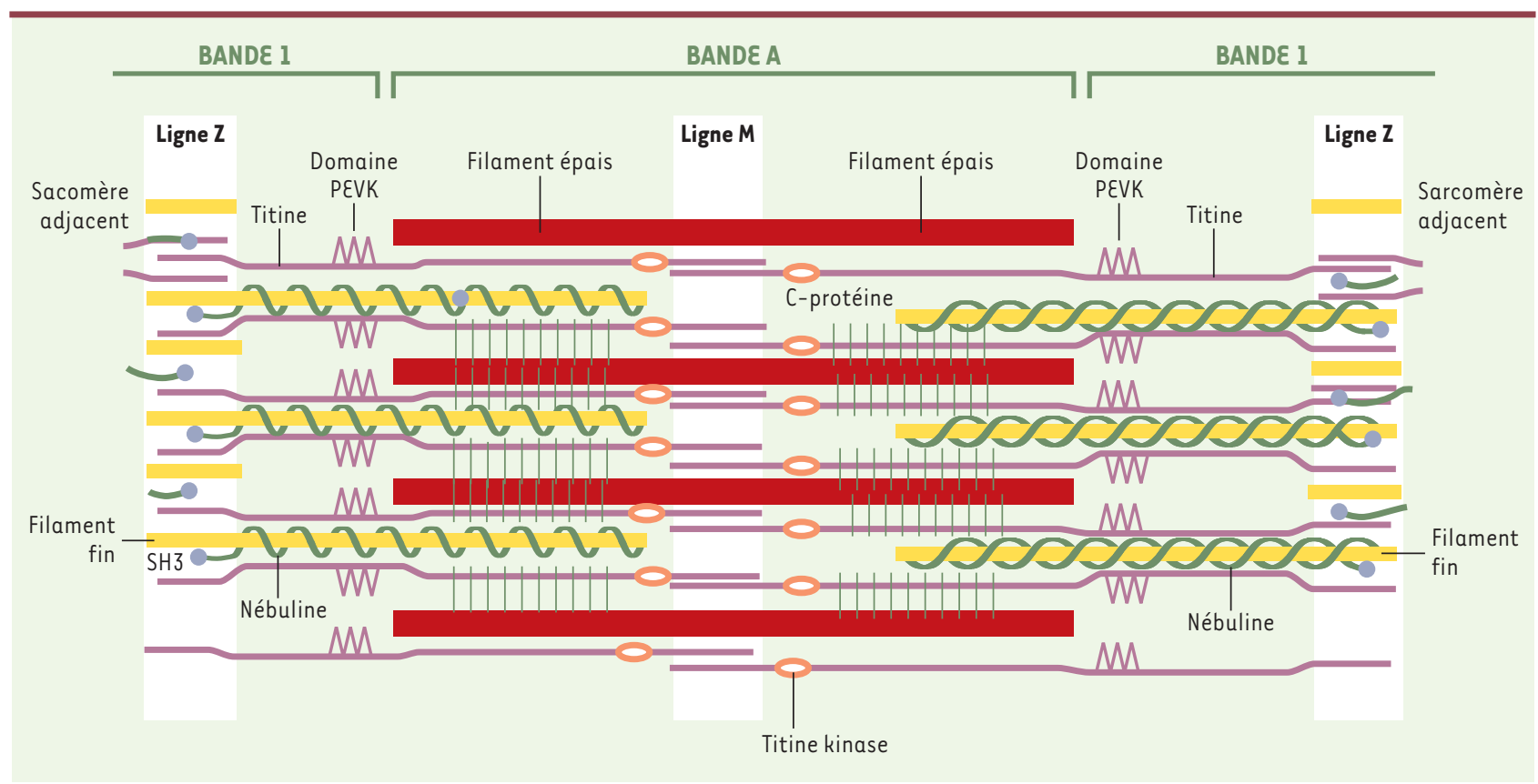

Figure 1. Représentation schématique d'un sarcomère de muscle squelettique. On voit représentés quatre éléments constitutifs du sarcomère (ligament fin, ligament épais, titine et nébuline). Les lignes $Z$ sont recouvertes par les régions amino-terminales et les lignes $M$ par les régions carboxy-terminales des molécules de titine. Le segment PEVK est ainsi appelé en raison des acides aminés qui le constituent : Pro-Glu-Val-Lys. (d'après [6]). 
spécifique de la titine cardiaque (comme c'est le cas pour le mutant pik chez le poisson zèbre), et pourtant, contrairement à ce que l'on aurait pu attendre, surtout pour la mutation tronquante, on n'observe aucune atteinte du muscle squelettique. II faudra donc d'abord comprendre chez les malades les troubles fonctionnels de la protéine tronquée. Elle devrait avoir perdu le domaine kinase de la tinine, et les sites de liaison aux filaments épais et à la calpaïne 3 entre autres.

Ensuite, il conviendra d'analyser la titine dans les autres familles de CMDIG, afin de retrouver sans doute d'autres mutations. Ainsi nous pour- rons connaître le rôle précis de la titine dans l'assemblage des myofibrilles et commencer à explorer les divers mécanismes pathogéniques responsables chez l'homme de cardiomyopathies dilatées. $\bullet$

\section{Mutations of TTN cause}

cardiomyopathy

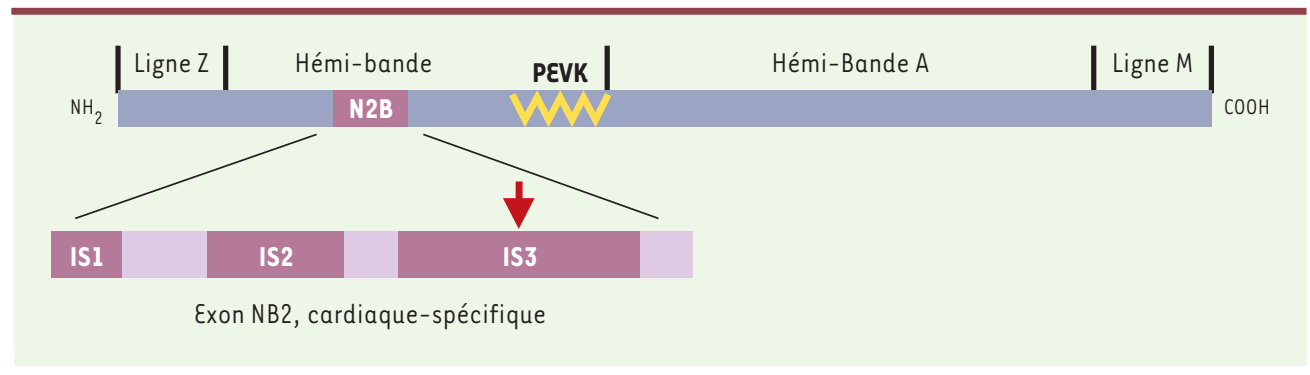

Figure 2. Représentation modulaire de la titine cardiaque codée par ttn, le gène de la mutation pickwick chez le poisson zèbre. L'exon N2B est localisé dans la bande I (domaine Ig) et contient 3 séquences uniques ISI, IS2, IS3. La flèche rouge indique la position de la mutation identifiée chez le poisson Pik ${ }^{\mathrm{ml7}}$ (d'après [3]).

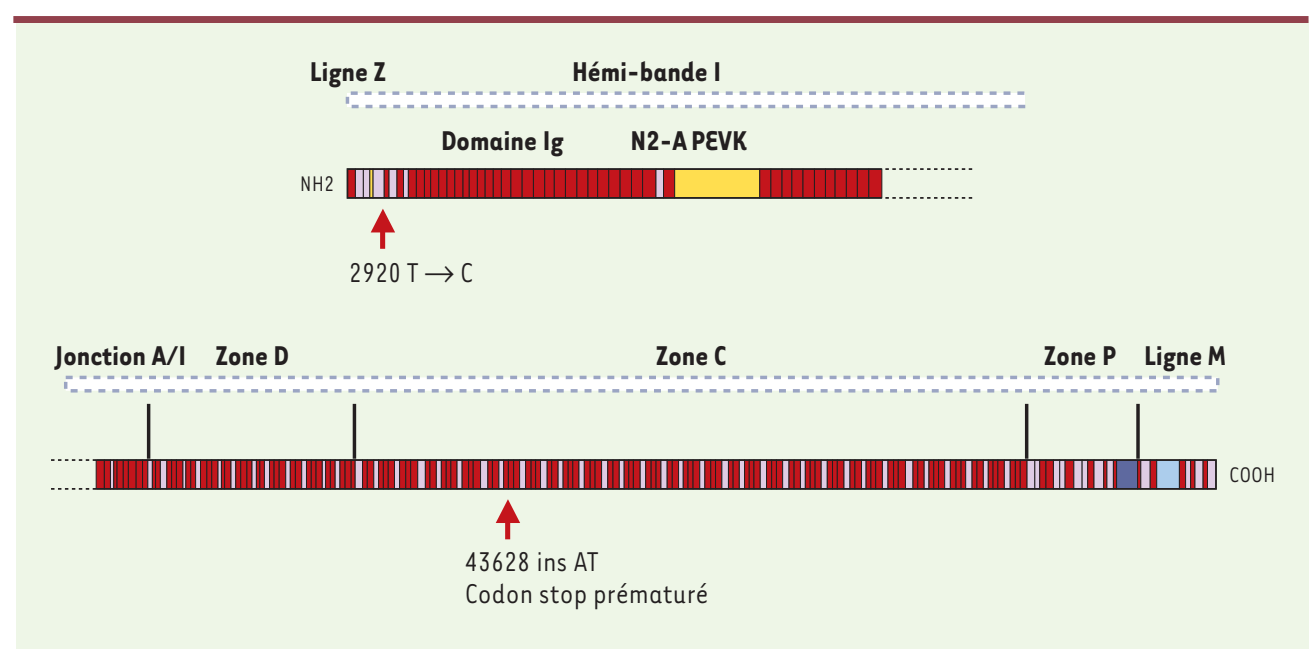

Figure 3. Représentation modulaire d'un filament de titine avec la localisation des mutations trouvées dans deux cardiopathies dilatées CMDIG (d'après [8]).

\section{RÉFÉRENCES}

1. Labeit S, Kolmerer B. Titins : giant proteins in charge of muscle ultrastructure and elasticity. Science 1995 ; $270: 293-6$.

2. Satoh MM, Takahashi M, Sakamoto T, Hiroe $M$ Marumo F, Kimura A. Structural analysis of the titin gene in hypertrophic cardiomyopathy : identification of a novel disease gene. Biochem Biophys Res Commun $1999 ; 262$ : 411-7.

3. Xu X, Meller SE, Zhong TP, et al. Cardiomyopathy in zebrafish due to mutation in an alternatively spliced exon of titin. Nat Genet $2002 ; 30: 205-9$.

4. Siu BL, Niimura $H$, Osborne JA, et al. Familial dilated cardiomyopathy locus maps to chromosome 2q31. Circulation $1999 ; 99$. 1022-6.

5. Person V, Kostin S, Susuki K, Labeit S, Schaper J.

Antisense oligonucleotide experiments elucidate the essential role of titin in sarcomerogenesis in adult rat cardiomyocytes in log-term culture. J Cell Sci 2000 ; 113 ; 3851-9.

6. Zhang $Y$, Featherstone $D$, Davis W, Rushton $\varepsilon$, Broadie K. Drosophila D-titin is required for myoblast fusion and skeletal muscle striation. J Cell Sci 2000 ; 113 : 3103-15.

7. Gerull B, Gramlich M, Atherton J, et al. Mutations of $T T N$,

encoding the giant muscle filament titin, cause familial dilated cardiomyopathy. Nat Genet $2002 ; 30$ : 201-4

8. Gregorio CC, Granzier H, Sorimachi H, Labeit $\mathrm{S}$. Muscle assembly : a titanic achievement? Curr Opin Cell Biol 1999 ; 11 : 18-25.

9. Kamisago M, Sharma SD, DePalma SR, et al. Mutations in sarcomere protein genes as a cause of dilated cardiomyopathy. $N$ Eng J Med 2000 ; 343 : 1688-96. 